\title{
NeuroImage
}

\section{Caffeine alters the temporal dynamics of the visual BOLD response}

\author{
Thomas T. Liu, ${ }^{\text {a,* }}$ Yashar Behzadi, ${ }^{\text {a,b }}$ Khaled Restom, ${ }^{a}$ Kamil Uludag, ${ }^{a}$ Kun Lu, \\ Giedrius T. Buracas, ${ }^{\mathrm{a}}$ David J. Dubowitz, ${ }^{\mathrm{a}}$ and Richard B. Buxton ${ }^{\mathrm{a}}$ \\ ${ }^{a}$ Center for Functional Magnetic Resonance Imaging and Department of Radiology, University of California San Diego, La Jolla CA, USA \\ ${ }^{\mathrm{b}}$ Department of Bioengineering, University of California San Diego, La Jolla, CA, USA
}

Received 6 April 2004; revised 19 July 2004; accepted 22 July 2004

\begin{abstract}
The blood oxygenation level-dependent (BOLD) responses to visual stimuli, using both a 1-s long single trial stimulus and a 20-s long block stimulus, were measured in a 4-T magnetic field both before and immediately after a 200-mg caffeine dose. In addition, resting levels of cerebral blood flow (CBF) were measured using arterial spin labeling. For the single trial stimulus, the caffeine dose significantly $(p<0.05)$ reduced the time to peak (TTP), the time after the peak at which the response returned to $50 \%$ of the peak amplitude $\left(\mathrm{TA}_{50}\right)$, and the amplitude of the poststimulus undershoot in all subjects $(N=5)$. Other parameters, such as the full-width half-maximum (FWHM) and the peak amplitude, also showed significant changes in the majority of subjects. For the block stimulus, the TTP, $\mathrm{TA}_{50}$, and the time for the response to reach $50 \%$ of the peak amplitude $\left(T_{50}\right)$ were significantly reduced. In some subjects, oscillations were observed in the poststimulus portion of the response with median peak periods of 9.1 and $9.5 \mathrm{~s}$ for the single trial and block responses, respectively. Resting CBF was reduced by an average of $\mathbf{2 4 \%}$. The reproducibility of the results was verified in one subject who was scanned on 3 different days. The dynamic changes are similar to those previously reported for baseline CBF reductions induced by hypocapnia and hyperoxia.

(C) 2004 Elsevier Inc. All rights reserved.
\end{abstract}

Keywords: BOLD responses; Hypocapnia; Hyperoxia

\section{Introduction}

The blood oxygenation level-dependent (BOLD) signal measured in functional magnetic resonance imaging (fMRI) experiments is a complex function of cerebral blood flow (CBF), cerebral blood volume, and oxygen metabolism (Buxton et al., 1998). The shape of the BOLD hemodynamic response (HDR) exhibits

* Corresponding author. UCSD Center for Functional MRI, Department of Radiology, 9500 Gilman Drive, MC 0677, La Jolla, CA 920930677. Fax: +18588220605 .

E-mail address: ttliu@ucsd.edu (T.T. Liu).

Available online on ScienceDirect (www.sciencedirect.com.) significant variability between subjects and somewhat less variability in repeated scans of a single subject (Aguirre et al., 1998; D’Esposito et al., 2003; Handwerker et al., 2004). This variability in the BOLD temporal dynamics has a direct impact on the interpretation of fMRI experiments, especially for event-related experiments in which the shape of the HDR is often of interest (Rosen et al., 1998). An understanding of the factors that contribute to the variability of the HDR is therefore important when comparing responses between populations or experimental conditions.

Recent studies have suggested that the temporal dynamics of the BOLD response are strongly dependent on the baseline CBF state. Kemna and Posse (2001) modified CBF levels by adjusting the arterial partial pressure of carbon dioxide $\left(\mathrm{PaCO}_{2}\right)$ with either controlled hyperventilation (hypocapnia) or $\mathrm{CO}_{2}$ breathing (hypercapnia). Using a 1.5-T system, they found that the temporal width and time to peak (TTP) of the BOLD response to a short visual stimulus increased with the baseline level of $\mathrm{PaCO}_{2}$. In a later study at $7 \mathrm{~T}$, Cohen et al. (2002) observed a similar dependence on $\mathrm{PaCO}_{2}$ and noted that the peak amplitude of the visual BOLD signal decreased with hypercapnia and increased with hypocapnia. In addition, the poststimulus undershoot in the response resolved more quickly with hypocapnia and appeared to be abolished with hypercapnia.

The carbon dioxide studies suggest that similar dynamic effects should be observed with other vasoactive agents that modify the baseline CBF state. One such agent is caffeine, which is the most widely consumed neural stimulant in the world with most of the intake coming from dietary sources such as coffee and tea (Fredholm et al., 1999). In addition to its neurostimulant effects, caffeine acts to reduce $\mathrm{CBF}$, primarily by inhibition of the adenosine $\mathrm{A}_{2 \mathrm{~A}}$ receptors (Cameron et al., 1990; Ngai et al., 2001). Several studies have examined the effect of caffeine on the amplitude of the BOLD response. In a study using a 1.5-T system, Mulderink et al. (2002) found that a 200-mg caffeine dose increased the amplitude of the BOLD response to a brief 2-s stimulus by $37 \%$ and $26 \%$ in the visual and motor cortices, respectively, and suggested that caffeine could be used as a contrast booster for BOLD studies. Laurienti et al. (2002, 2003), however, 
found that the BOLD response to a 30 -s-long visual stimulus was not increased uniformly in all subjects and also observed a dependence on chronic caffeine usage. The discrepancies in the studies may have been due to the difference in stimulus duration and intrinsic variation in the subject populations. Aside from the amplitude changes, neither study examined changes in the temporal dynamics of the BOLD response, which may have also affected the analysis. For example, a change in the shape of the hemodynamic response, such as a decrease in the temporal width, would affect the correlation with a canonical reference function.

In this study, we examined the effect of a 200-mg caffeine dose on the temporal dynamics of the BOLD response to visual stimuli of both short (1 s) and long (20 s) duration. In addition, we used arterial spin labeling (ASL) to noninvasively measure the effect of caffeine on baseline CBF.

\section{Methods}

\section{Experimental protocol}

Six healthy adult male volunteers (ages 23 to 40 years) participated in the study after giving informed consent. Each subject was asked to refrain from ingesting any food or drink containing caffeine for at least $12 \mathrm{~h}$ before the imaging session. The estimated daily caffeine usage of the subjects based on selfreports of coffee, tea, and caffeinated soda consumption is shown in Table 1. The assumed caffeine contents for an 8-oz cup of coffee, an 8-oz cup of tea, and a 12-oz can of soda were $100 \mathrm{mg}, 40$ $\mathrm{mg}$, and $20 \mathrm{mg}$, respectively (Fredholm et al., 1999).

Each imaging session lasted for approximately $3.5 \mathrm{~h}$ and consisted of a 1.5 -h predose section followed by a 1.5 -h postdose section. Between sections, the subject ingested an over-the-counter tablet containing $200 \mathrm{mg}$ of caffeine and rested outside of the magnet for 20 to $30 \mathrm{~min}$. The first imaging run of the postdose section started approximately $40 \mathrm{~min}$ after the dose. This interval was chosen based on studies showing that the absorption of caffeine from the gastrointestinal tract reaches $99 \%$ about $45 \mathrm{~min}$ after ingestion, with a half-life ranging from 2.5 to $4.5 \mathrm{~h}$ (Fredholm et al., 1999). During each imaging section, the subject was presented with a visual stimulus consisting of "on" periods of a full-field, full-contrast radial $8-\mathrm{Hz}$ flickering checkerboard with a small white fixation square at the center of the screen and "off" periods of a black background with a white fixation square. Each imaging section consisted of the following sets of paradigms: (a) three repeats of a block design paradigm with a 20-s initial off period followed by four cycles of 20-s on and 40-s off, (b) three repeats of a periodic single trial paradigm with an initial 12 -s off period followed by 8 cycles of 1-s on and 30-s off, and (c) three additional repeats of the block design paradigm for the acquisition of functional perfusion data using arterial spin labeling.

To assess repeatability, subject 1 was studied on 3 different days (days 1, 8, and 10) using the same imaging protocol on each day. Data for this subject are denoted as $1 \mathrm{a}, 1 \mathrm{~b}$, and $1 \mathrm{c}$ for days 1,8 , and 10 , respectively. This subject had no caffeine for at least $36 \mathrm{~h}$ prior to each session.

\section{Image acquisition}

Imaging data were acquired on a 4-T whole body system (Varian Inc., Palo Alto, CA) with a head transmit coil and a surface receive coil (Nova Medical, Wakefield, MA) placed next to the subject's occipital lobe. Laser alignment was used to landmark the patient for each imaging section and minimize differences in head position between sections. During the first block design paradigm, data were acquired with a PICORE (Wong et al., 1997) arterial spin labeling (ASL) sequence $(\mathrm{TR}=2 \mathrm{~s}$, TI $=1100 \mathrm{~ms}, 10-\mathrm{cm}$ tag width, and a 1-cm tag-slice gap) with echoplanar imaging (EPI) readout $\left(\mathrm{TE}=26 \mathrm{~ms}\right.$, flip angle $=90^{\circ}$, FOV $24 \mathrm{~cm}, 64 \times 64$ matrix). Six 4-mm-thick oblique slices aligned with the calcarine sulcus were acquired in a linear fashion from inferior to superior. This run was used to verify functional activation in the prescribed slices but was not otherwise used for the analysis presented in this paper. For the periodic single trial runs and the second and third block designs runs, BOLD-weighted images were acquired with an EPI sequence with $\mathrm{TR}=1 \mathrm{~s}, \mathrm{TE}=26 \mathrm{~ms}$, flip angle $70^{\circ}, \mathrm{FOV}$ $24 \mathrm{~cm}, 64 \times 64$ matrix, six interleaved slices.

Quantitative ASL images were acquired with the head coil in transmit and receive mode and a PICORE QUIPSS II (PQ2) sequence with $\mathrm{TR}=2 \mathrm{~s}, 130$ repetitions, TI $=700 \mathrm{~ms}$, TI $2=1400$ $\mathrm{ms}, 10-\mathrm{cm}$ tag width, $1-\mathrm{cm}$ tag to proximal slice gap, and the same in-plane EPI parameters as the PICORE run (Wong et al., 1998). Three 8-mm slices were acquired to cover the same volume as the six 4-mm slices acquired in the PICORE and BOLD runs. The subject viewed the dark screen with a white fixation cross. Proton density and $\mathrm{T}_{2}{ }^{*}$-weighted EPI images (referred to as $M_{0}+T_{2}{ }^{*}$ images) with the same in-plane parameters as the PQ2 runs but with a TR of $20 \mathrm{~s}$ were acquired with both volume and surface coils. These long TR scans were necessary because the first image acquired in each scan was a phase map used to correct the remaining images. In addition to the baseline scans, three block runs with ASL scans were also performed but are not analyzed in this paper.

High-resolution structural scans were acquired with a magnetization-prepared 3D fast low angle shot (3D MP-FLASH) sequence

Table 1

Predose and postdose baseline CBF values shown as mean (standard deviation)

\begin{tabular}{lllll}
\hline Subject & Estimated daily caffeine usage $(\mathrm{mg})$ & Predose baseline CBF ml/(100 g min) & Postdose baseline CBF ml/(100 g min) & Paired $t$ test $p$-value \\
\hline $1 \mathrm{a}$ & $<50$ & $76.1(34.2)$ & $46.6(28.5)$ & $7.0 \times 10^{-6}$ \\
$1 \mathrm{~b}$ & $<50$ & $68.3(33.6)$ & $41.1(29.8)$ & $4.0 \times 10^{-7}$ \\
$1 \mathrm{c}$ & $<50$ & $61.0(33.8)$ & $37.9(29.5)$ & $1.0 \times 10^{-10}$ \\
2 & 200 & $80.0(31.4)$ & $60.0(24.0)$ & $5.0 \times 10^{-5}$ \\
3 & 200 & $99.7(31.1)$ & $78.2(33.1)$ & $4.9 \times 10^{-5}$ \\
4 & 160 & $65.7(30.7)$ & $54.3(22.6)$ & $2.1 \times 10^{-3}$ \\
5 & $<50$ & $68.6(36.2)$ & $54.5(31.3)$ & $3.1 \times 10^{-3}$ \\
\hline
\end{tabular}

For each subject, significance was computed with a paired $t$ test. 
with the following parameters: four phase encode interleaves, $\mathrm{TR}=$ $10.5 \mathrm{~ms}, \mathrm{TE}=4.8 \mathrm{~ms}$, flip angle $=11^{\circ}, 256 \times 256 \times 192 \mathrm{~mm}$, FOV $256 \times 256 \times 128$ matrix.

\section{Retinotopic maps}

An additional set of scanning sessions was used to define a retinotopic region of interest (ROI) for each subject. Imaging was performed on a 1.5-T whole body system (Siemens, Erlangen, Germany). A high-resolution whole-brain structural scan (3D MPFLASH acquired with birdcage head coil; 1-mm isotropic resolution) was acquired for segmentation of cortical gray matter (Teo et al., 1997). The segmented cortical gray matter of occipital poles was then flattened using surface rendering methods described in Wandell et al. (2000). Functional scans for mapping retinotopic organization of early visual areas were acquired using the standard visual stimuli for retinotopic mapping (Engel et al., 1994; Press et al., 2001), a small surface coil placed under the subject's head, and an EPI sequence with the following parameters: $\mathrm{TR}=2 \mathrm{~s}, \mathrm{TE}=40 \mathrm{~ms}$, flip angle $70^{\circ}$, matrix $64 \times 64,3-\mathrm{mm}$ isotropic resolution. At the end of the session, an additional highresolution anatomical scan (1-mm isotropic resolution) was acquired with the surface coil. This anatomical scan was used to align the retinotopic data with the high-resolution structural scan used for flattening of cortical surfaces. Linear trends were removed from the functional scans. Activation was assessed by correlating detrended data with the first harmonic of the stimulus variation frequency (Press et al., 2001). Representation of the primary visual area V1 of each subject was delineated on the computationally flattened visual cortex to define the retinotopic ROI for each subject.

\section{Data analysis}

All images were coregistered using AFNI software (Cox, 1996). The structural scan from each postdose session was aligned to the structural scan of its respective predose session, and the rotation and shift parameters used for this alignment were then applied to the postdose functional (BOLD and ASL) images. In a similar fashion, the rotation and shift parameters used to register each retinotopic structural scan with the corresponding predose structural scan were applied to the retinotopic ROIs. Images from scans of subject 1 acquired on days 8 and 10 were aligned to that subject's day 1 predose session.

For the BOLD runs, average responses for the block and single trial designs were formed by averaging over all runs, experimental cycles, and voxels in the retinotopic ROI. Data from subject 6 exhibited severe motion artifacts and low signal-to-noise ratios and were not further analyzed. In addition, the first single trial run for subject 5 was not used in the averaging because of severe motion artifacts. Each subject's average responses were interpolated to a time resolution of $0.1 \mathrm{~s}$ and the following eight parameters were calculated: (1) time to reach $10 \%$ of the peak response $\left(\mathrm{T}_{10}\right),(2)$ time after the peak at which the response returned to $10 \%$ of the peak response $\left(\mathrm{TA}_{10}\right),(3)$ time to $50 \%$ of the peak response $\left(\mathrm{T}_{50}\right)$, (4) time after the peak at which the response returned to $50 \%$ of the peak response $\left(\mathrm{TA}_{50}\right)$, (5) full-width half-maximum $(\mathrm{FWHM}=$ $\mathrm{TA}_{50}-\mathrm{T}_{50}$ ), (6) peak amplitude, (7) time to peak, and (8) amplitude of the poststimulus undershoot response. Differences in these parameters due to the caffeine dose were assessed with a paired $t$ test.
To assess the variability of the responses across voxels in the retinotopic ROI, correlation analysis was used to select voxels with sufficient signal-to-noise ratio to make the parameter measurements. The responses for each voxel were correlated with the corresponding average response for each condition and experimental design (e.g., average predose block response). For each subject, correlation thresholds were chosen to achieve an overall probability of false alarm of 0.01 for the voxel responses associated with each experimental design. For all voxels passing the threshold test, the eight timing and amplitude parameters described above were measured for each combination of design and condition. Differences in parameters due to the caffeine dose were assessed with a paired $t$ test.

Poststimulus responses were defined as the final 20 and $32 \mathrm{~s}$ of the periodic single trial and block responses, respectively. After removal of the mean and a linear trend, each response was zeropadded to 128 points and then Fourier-transformed. The frequency of the peak of the frequency spectrum was recorded for each voxel and then inverted to yield the peak period. Since histograms of the peak periods showed significant nonnormality, the effect of caffeine on the peak period was assessed using both a paired $t$ test and a Wilcoxon signed rank test.

To estimate baseline CBF, the average ASL difference signal $(\Delta M=$ control - tag $)$ was computed for each voxel in the retinotopic ROI after excluding the first nine time points. This signal was converted into the standard physiological units of $\mathrm{ml} /$ (100 g-min) with the formula $\mathrm{CBF}=\Delta M /\left(2 M_{0 A} T I_{1} \exp \left(-\mathrm{TI}_{2} /\right.\right.$ $\left.\mathrm{TI}_{1 B}\right) \exp \left(-\mathrm{TE} / \mathrm{T}_{2 B} *\right)$, where $C B F$ denotes cerebral blood flow, and $T_{2 B}{ }^{*}, T_{1 B}$, and $M_{0 A}$ are the apparent transverse relaxation time, longitudinal time constant, and equilibrium magnetization, respectively, of arterial blood (Wong et al., 1998). The value of $\mathrm{T}_{1 B}$ at $4 \mathrm{~T}$ was estimated to be $1600 \mathrm{~ms}$ based on published values at 1.5 and $3 \mathrm{~T}$ and a power law dependence of $\mathrm{T}_{1 B}$ on field strength (Lu et al., 2003a,b). The $M_{0}+T_{2}{ }^{*}$ EPI images were used as a rough estimate of $M_{0 A} \exp \left(-T E / T_{2 B} *\right)$, based on the similarity of the transverse relaxation times of arterial blood at $4.7 \mathrm{~T}$ and of tissue at $4 \mathrm{~T}$ (both around $33 \mathrm{~ms}$ ) (Gati et al., 1997; Silvennoinen et al., 2003) and the similarity of the proton densities of blood and tissue (Wong et al., 1998). Before their use for normalization, the $M_{0}+T_{2}$ * EPI images were smoothed with a Gaussian kernel with FWHM $=11.25 \mathrm{~mm}$. For each subject, the effect of caffeine on baseline CBF was assessed with a paired $t$ test.

\section{Results}

The group average predose and postdose BOLD responses are shown in Fig. 1. A qualitative assessment of the responses indicates that caffeine speeds up both the single trial and block responses and also appears to decrease the poststimulus undershoot amplitude of the single trial responses. A quantitative assessment of the single trial and block responses is provided in the scatter plots and paired $t$ test results shown in Figs. 2 and 3. When data from subject 1's second and third experimental sessions ( $1 \mathrm{~b}$ and $1 c)$ are not included, significant $(p<0.05)$ changes in $\mathrm{TA}_{50}$, time to peak, and undershoot amplitude are observed for the single trial responses, while significant changes in $\mathrm{T}_{10}, \mathrm{~T}_{50}, \mathrm{TA}_{50}$, and time to peak are observed for the block responses. For this and all subsequent analyses, probabilities ( $p$-values) were calculated assuming a null hypothesis of no significant difference in the means (i.e., two-tailed distribution). If the additional data on 
(a) Group Average Block Response

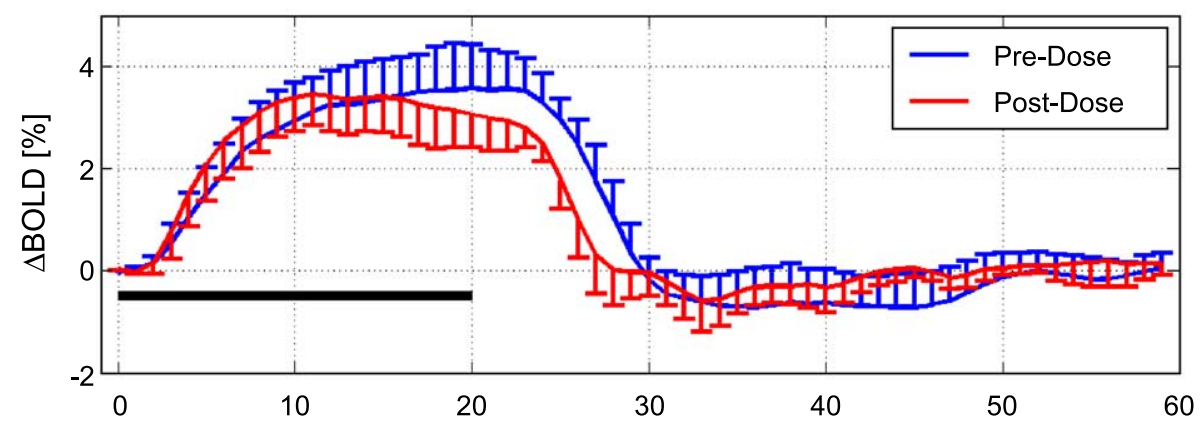

(b) Group Average Single Trial Response

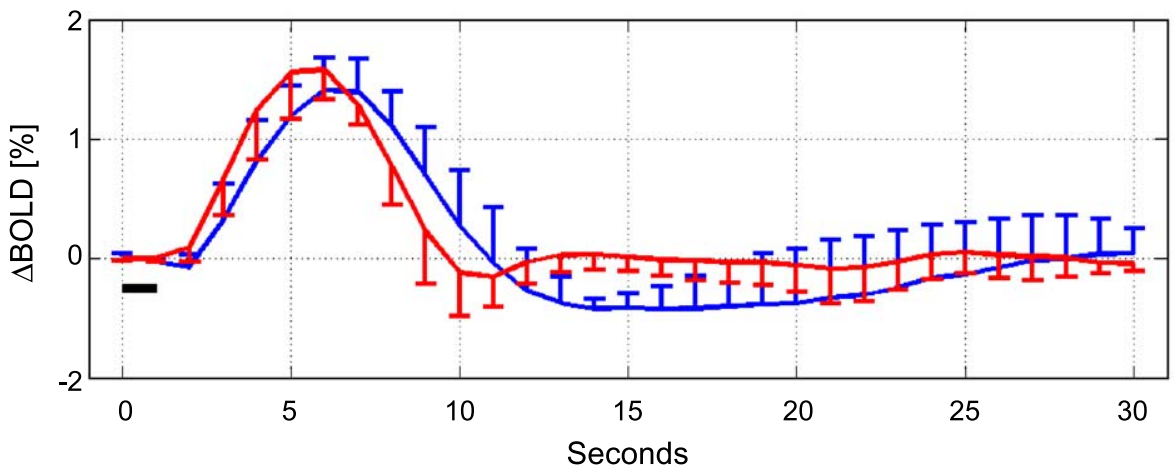

Fig. 1. Predose (blue) and postdose (red) single trial and block BOLD responses averaged over the first five subjects (response from day 1 was used for subject 1). The length of each error bar represents 1 standard deviation. The thick black bars denote the stimulus duration.

subject 1 are included, then changes in $\mathrm{T}_{10}, \mathrm{~T}_{50}$, and FWHM for the single trial responses become significant as well.

Examples of voxelwise variability in the parameters are shown in the scatter plots of Figs. 4 and 5 for the single trial and block responses, respectively, of subject 1a. For this subject, a significant $(p<0.005)$ difference is observed for all parameters of the single trial response and for all parameters except for FWHM and peak amplitude of the block response. Summary plots of the mean and

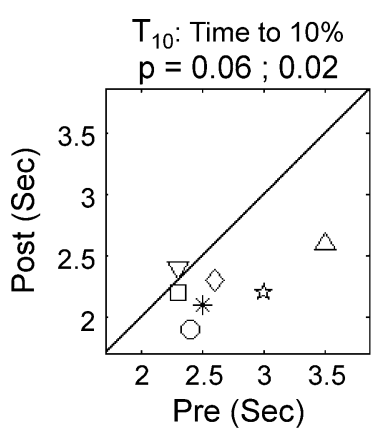

FWHM

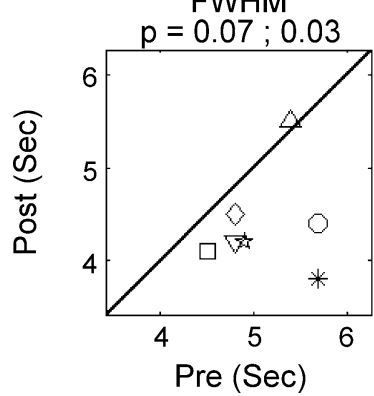

$\mathrm{TA}_{10}$ : Time to $10 \%$ After

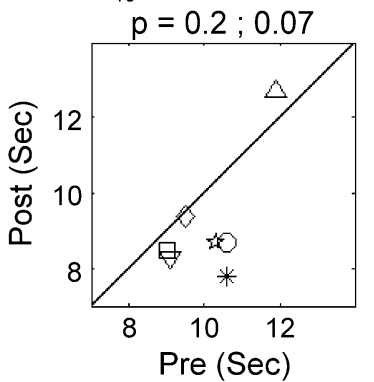

Peak Amplitude

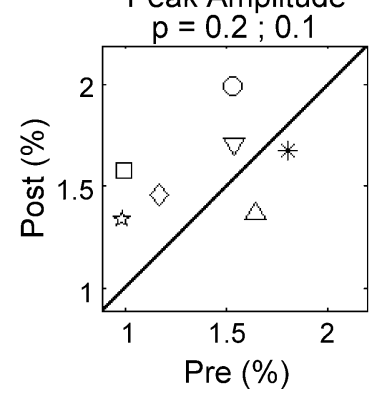

$\mathrm{T}_{50}$ : Time to $50 \%$
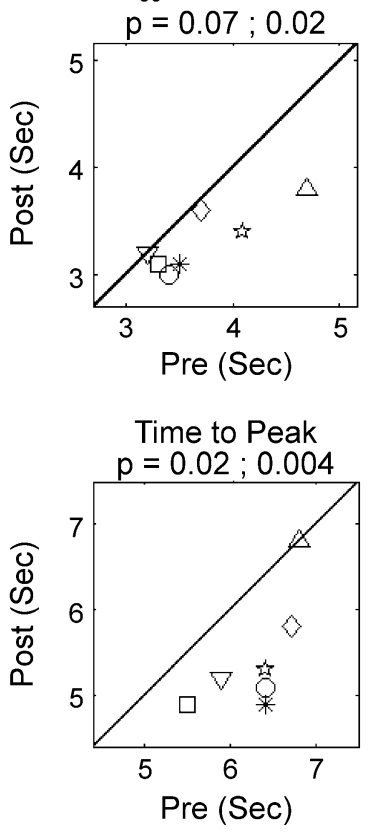

$\mathrm{TA}_{50}$ : Time to $50 \%$ After

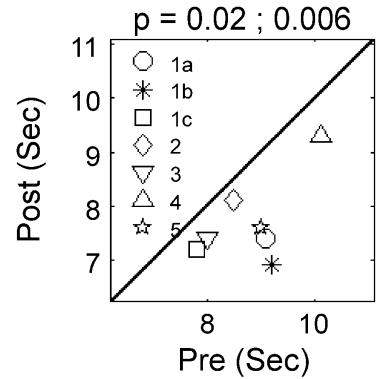

Undershoot Amplitude

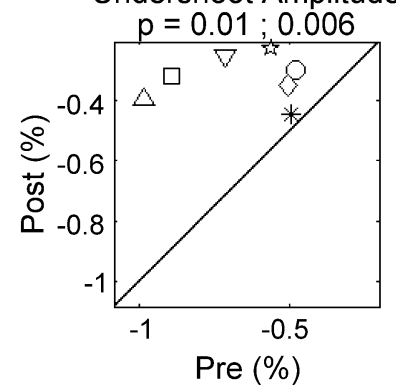

Fig. 2. Group scatter plots of timing and amplitude parameters computed from the ROI averaged periodic single trial responses for each subject. Probability values were obtained with a paired $t$ test. The first $p$-value listed includes data only from subject 1 's day 1 scan (data set 1a), while the second $p$-value listed includes parameters from all experiments on subject 1 (e.g., 1a, 1b, 1c). 


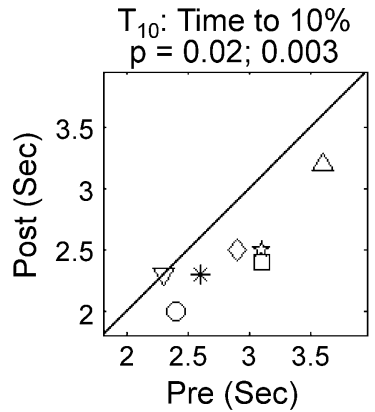

FWHM

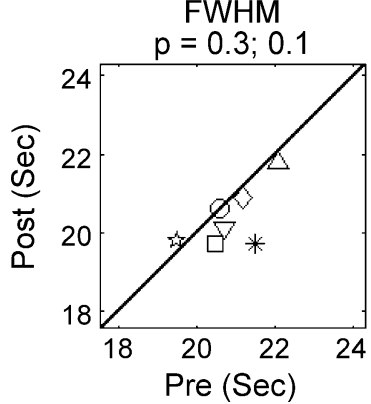

$\mathrm{TA}_{10}$ : Time to $10 \%$ After $p=0.5 ; 0.2$

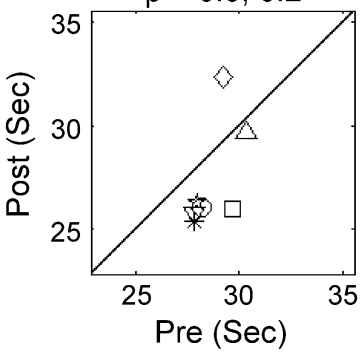

Peak Amplitude $\mathrm{p}=0.2 ; 0.06$

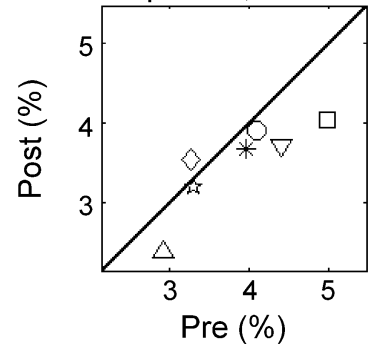

$\mathrm{T}_{50}$ : Time to $50 \%$

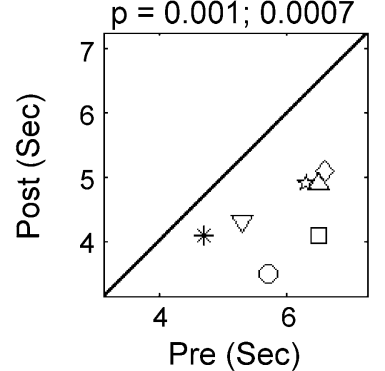

Time to Peak

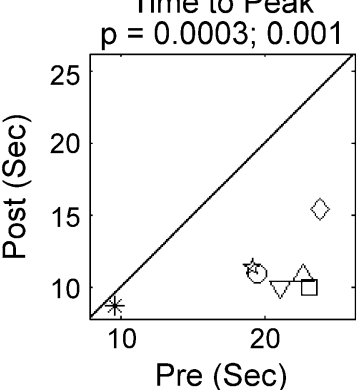

$\mathrm{TA}_{50}$ : Time to $50 \%$ After

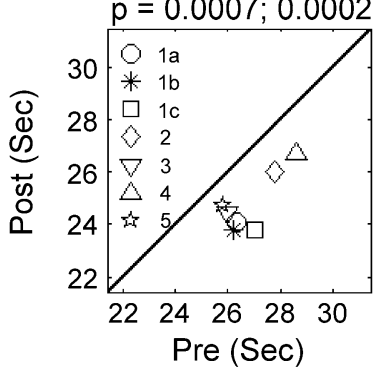

Undershoot Amplitude

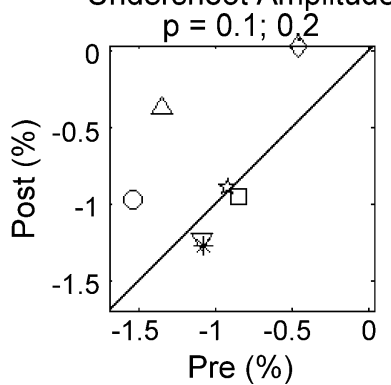

Fig. 3. Group scatter plots of timing and amplitude parameters computed from the ROI averaged block responses for each subject. Probability values were obtained with a paired $t$ test. The first $p$-value listed includes data only from subject 1's day 1 scan (data set 1a), while the second $p$-value listed includes parameters from all experiments on subject 1 (e.g., 1a, 1b, 1c).

standard deviation over voxels for each parameter and subject are shown in Figs. 6 and 7 with color coding to indicate the $p$-value for each subject. Note that since paired $t$ tests are used to assess significance, a small $p$-value can be attained even when the standard deviation is large if the predose and postdose parameters vary across voxels in a similar fashion. Consistent with the group data, all subjects showed a significant $(P<0.05)$ caffeine-related decrease in $\mathrm{TA}_{50}$, time to peak, and undershoot amplitude of the single trial response. In addition, all subjects showed significant differences in seven of the eight parameters, except for subject 3 who showed significant differences in five of the eight parameters. For the block responses, the parameters $\mathrm{T}_{50}, \mathrm{TA}_{50}$, and time to peak showed a significant $(p<0.05)$ caffeine-related decrease for all subjects.

Oscillations were observed in the poststimulus portion of the average responses in some subjects and were enhanced by the
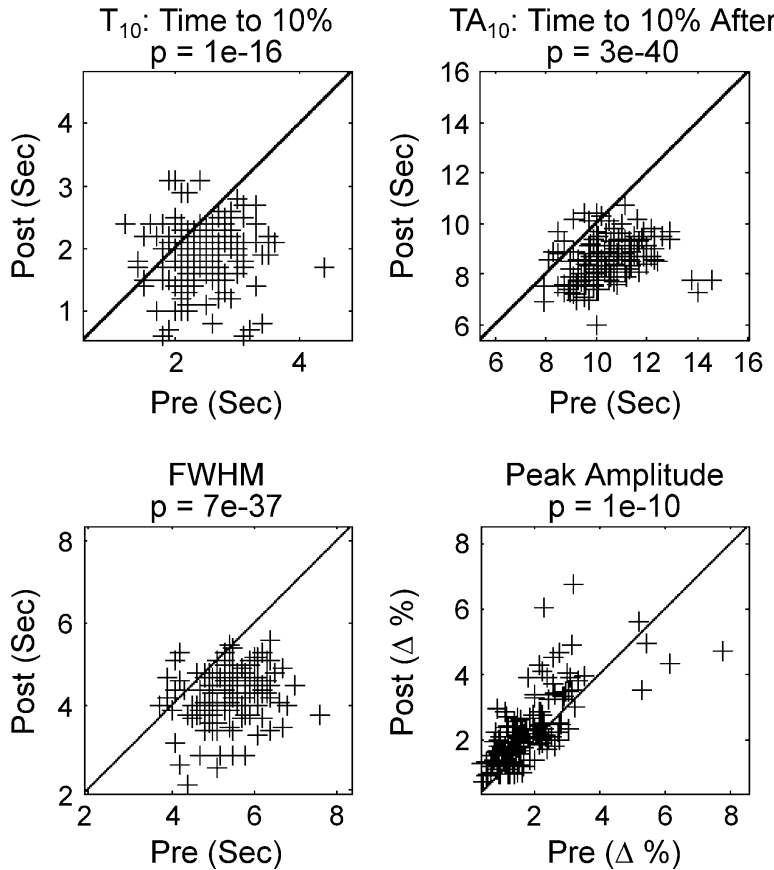

Peak Amplitude

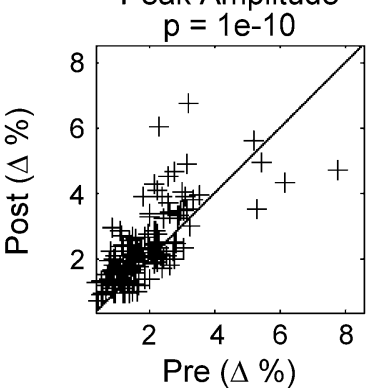

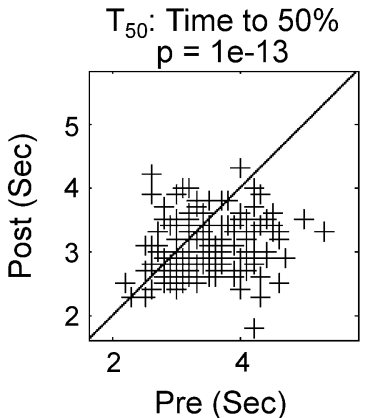

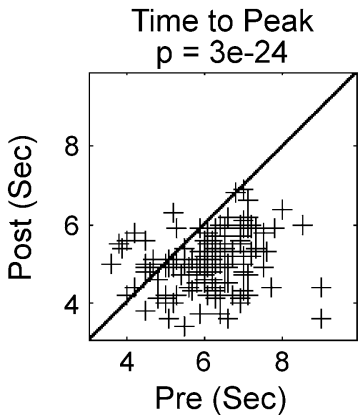

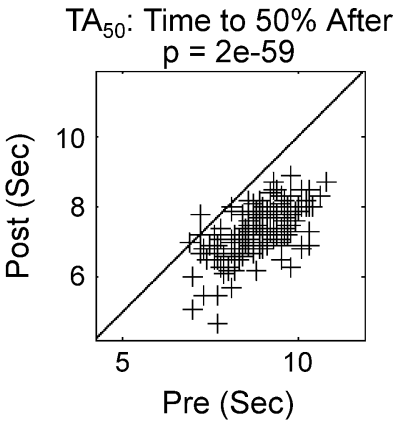

Undershoot Amplitude

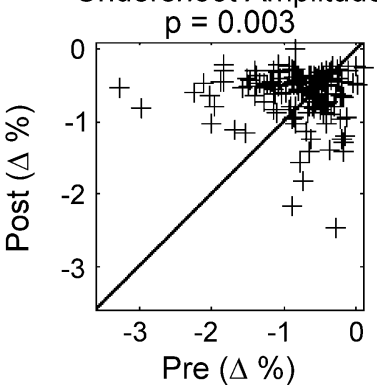

Fig. 4. Scatter plots of timing and amplitude parameters computed on a voxelwise basis for the periodic single trial responses of subject 1a. $p$-values were computed with a paired $t$ test. 

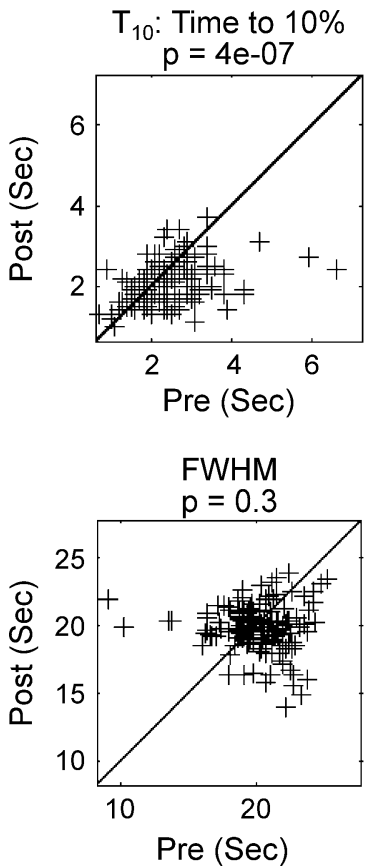
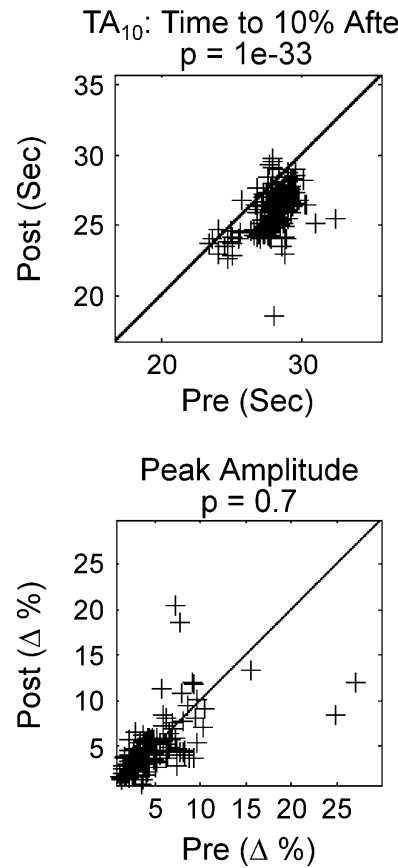

$\mathrm{T}_{50}:$ Time to $50 \%$ $p=4 e-27$
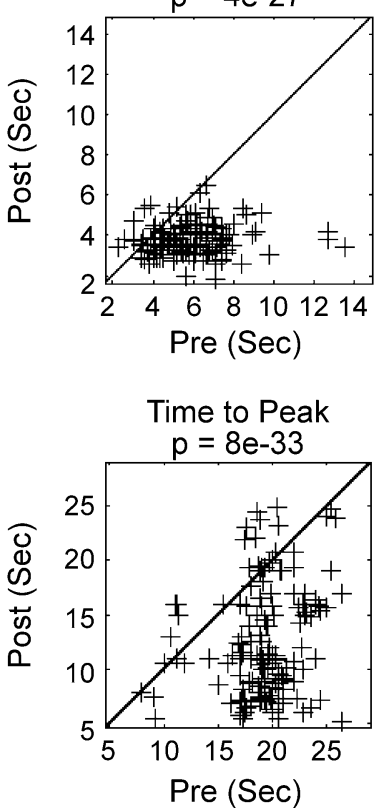

$\mathrm{TA}_{50}$ : Time to $50 \%$ After

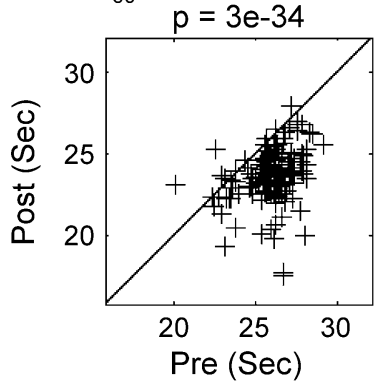

Undershoot Amplitude

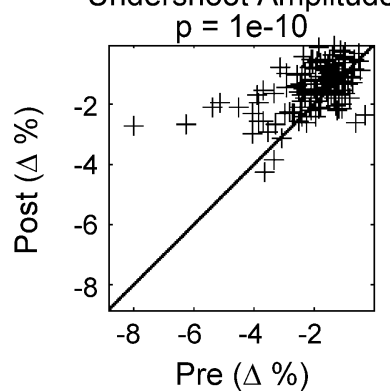

Fig. 5. Scatter plots of timing and amplitude parameters computed on a voxelwise basis for the block responses of subject 1a. $p$-values were computed with a paired $t$ test.

administration of the caffeine dose. Examples of oscillations in the poststimulus portion of the block response of subject 3 are shown in Fig. 8. Block responses for all voxels in the retinotopic ROI that passed the correlation threshold (see Methods section) were normalized by their peak amplitude, and the average normalized responses (mean \pm 1 standard deviation) are shown in the top row. The bottom row shows the responses corresponding to the 50 voxels that had the highest correlation coefficients. Both the mean responses and the individual voxel responses show a clear oscillation in the postdose condition. Similar oscillations are shown in the average responses from subject 1 shown in Fig. 11.

To further assess the oscillations, Fourier analysis (see Methods section) was used to assess the peak periods in the poststimulus portion of the single trial and block responses. Histograms of these peak periods across voxels are shown in Figs. 9 and 10 for each subject. For the single trial responses, all subjects except for subject 5 showed a significant $(p<0.002)$ caffeine-related decrease in the median peak period. Subjects 1 and 3 show a

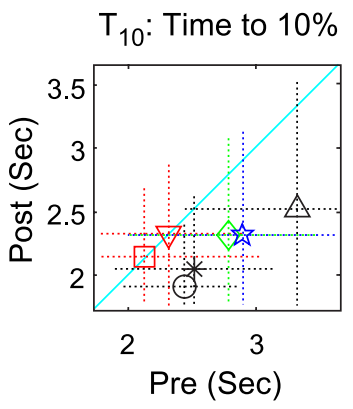

FWHM
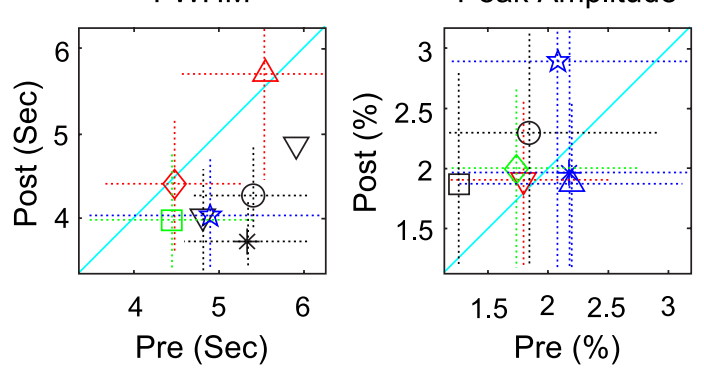

Not Significant

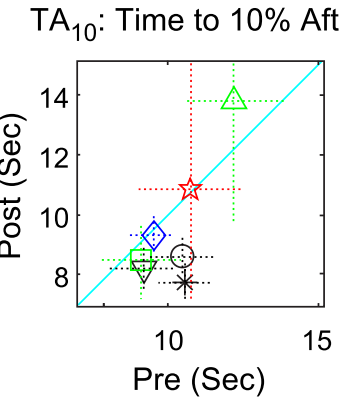

Peak Amplitude

$\mathrm{p}<0.05$

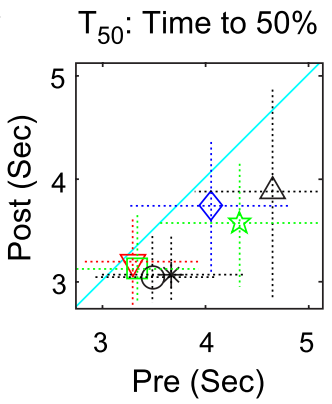

Time to Peak

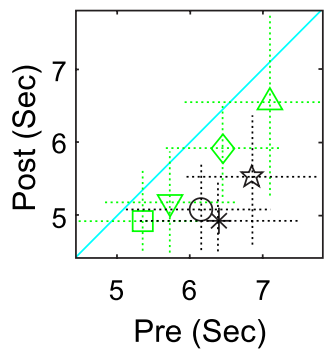

$\mathrm{p}<0.001$

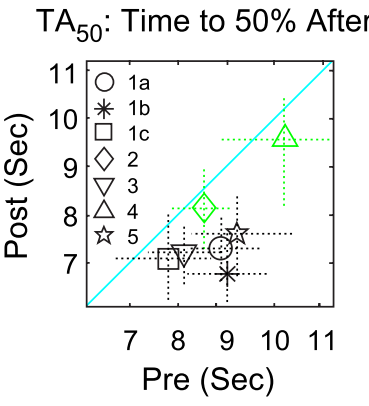

Undershoot Amplitude

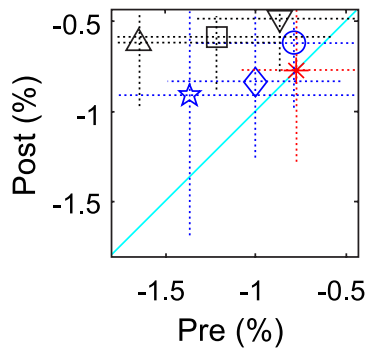

$p<1 e-6$

Fig. 6. Scatter plots of timing and amplitude parameters of periodic single trial responses for all subjects. For each subject, the mean value \pm 1 standard deviation of the parameters are color-coded by their significance level. 

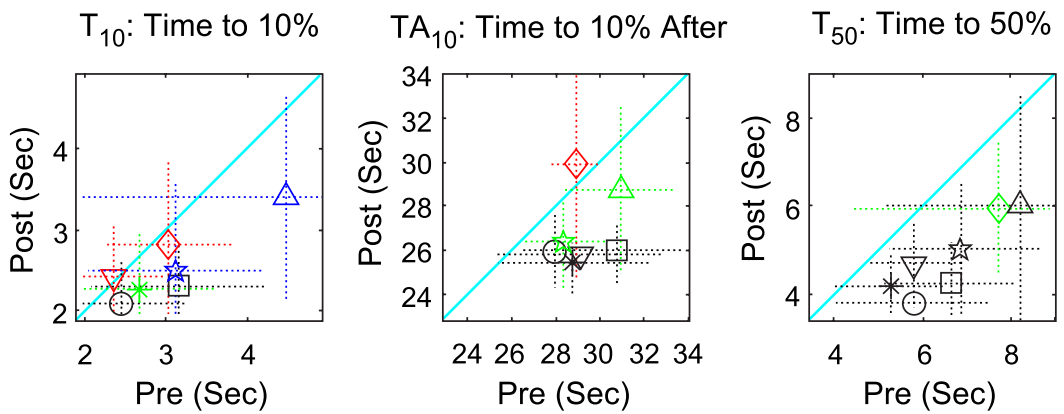

$\mathrm{TA}_{50}:$ Time to $50 \%$ After
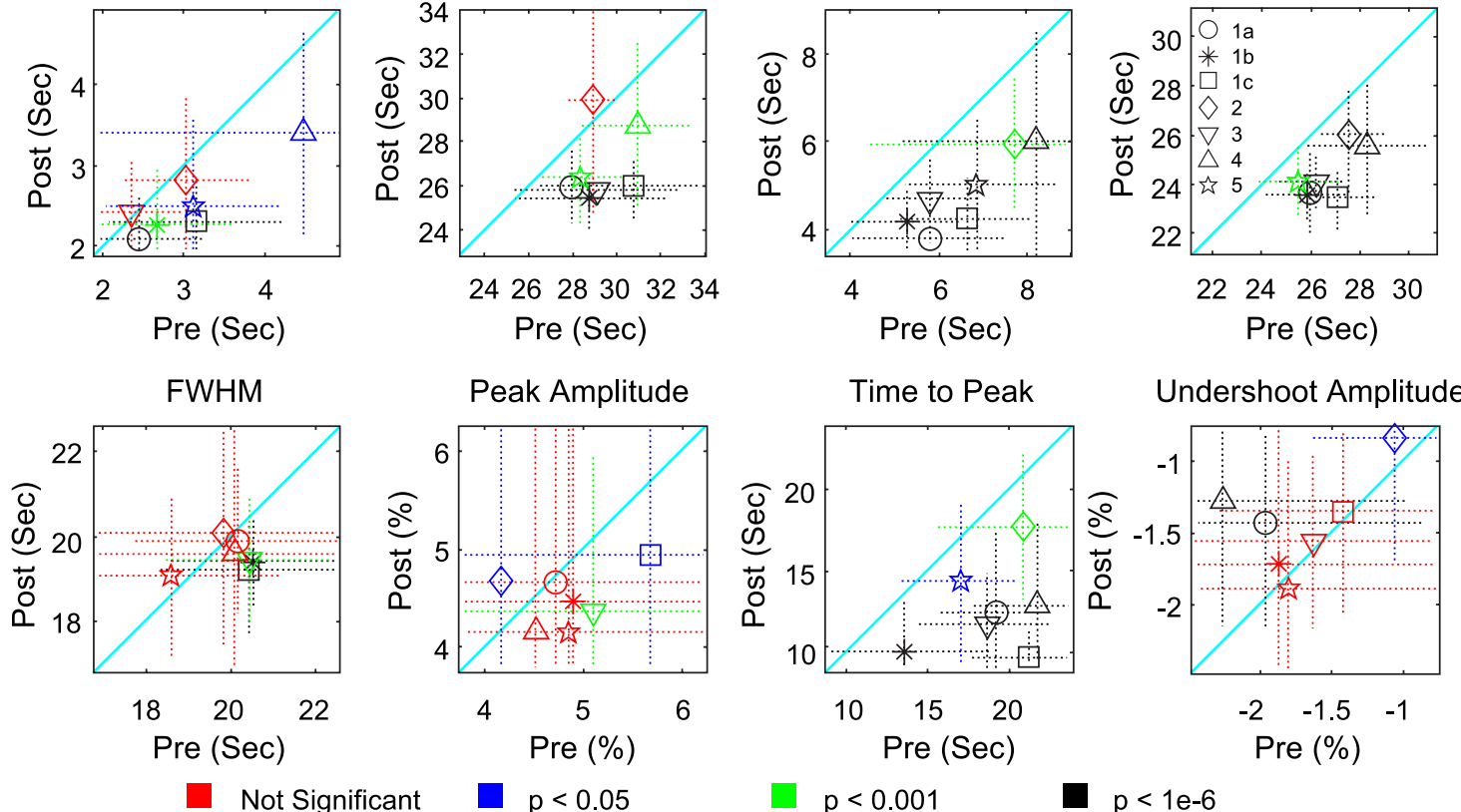

Undershoot Amplitude

- Not Significant

$\mathrm{p}<0.05$

$p<0.001$

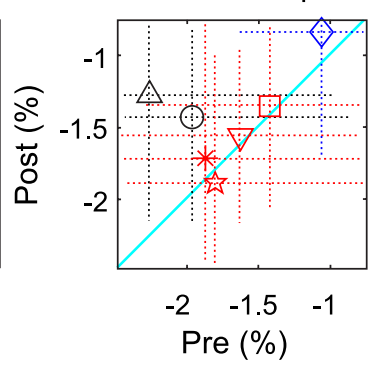

$p<1 e-6$

Fig. 7. Scatter plots of timing and amplitude parameters of block responses for all subjects. For each subject, the mean value \pm 1 standard deviation of the parameters are color-coded by their significance level.
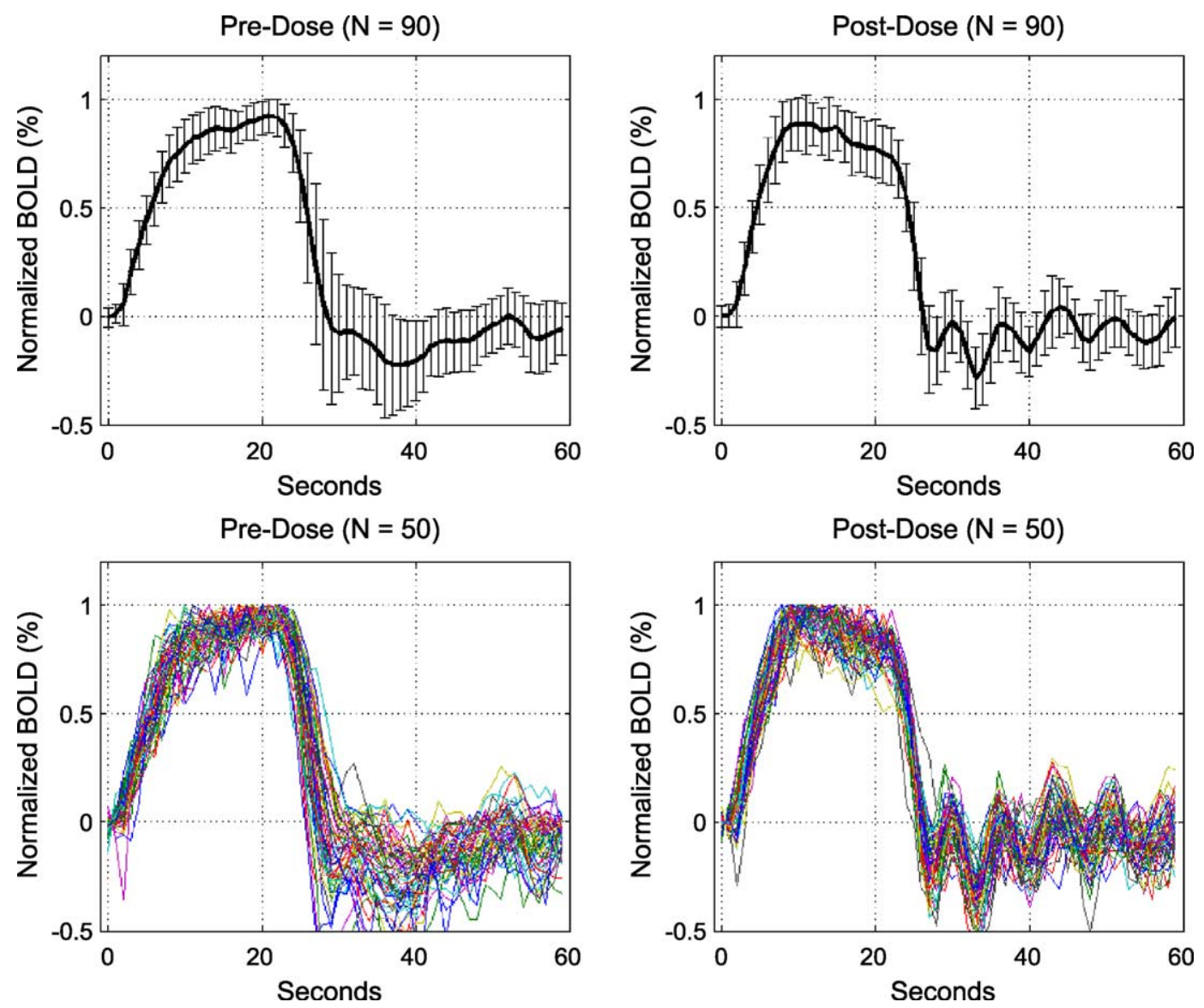

Fig. 8. Predose and postdose block responses for subject 3. The top row shows the mean \pm 1 standard deviation of the normalized responses (each voxel's response normalized by its peak value). The bottom row shows normalized responses from 50 different voxels. 

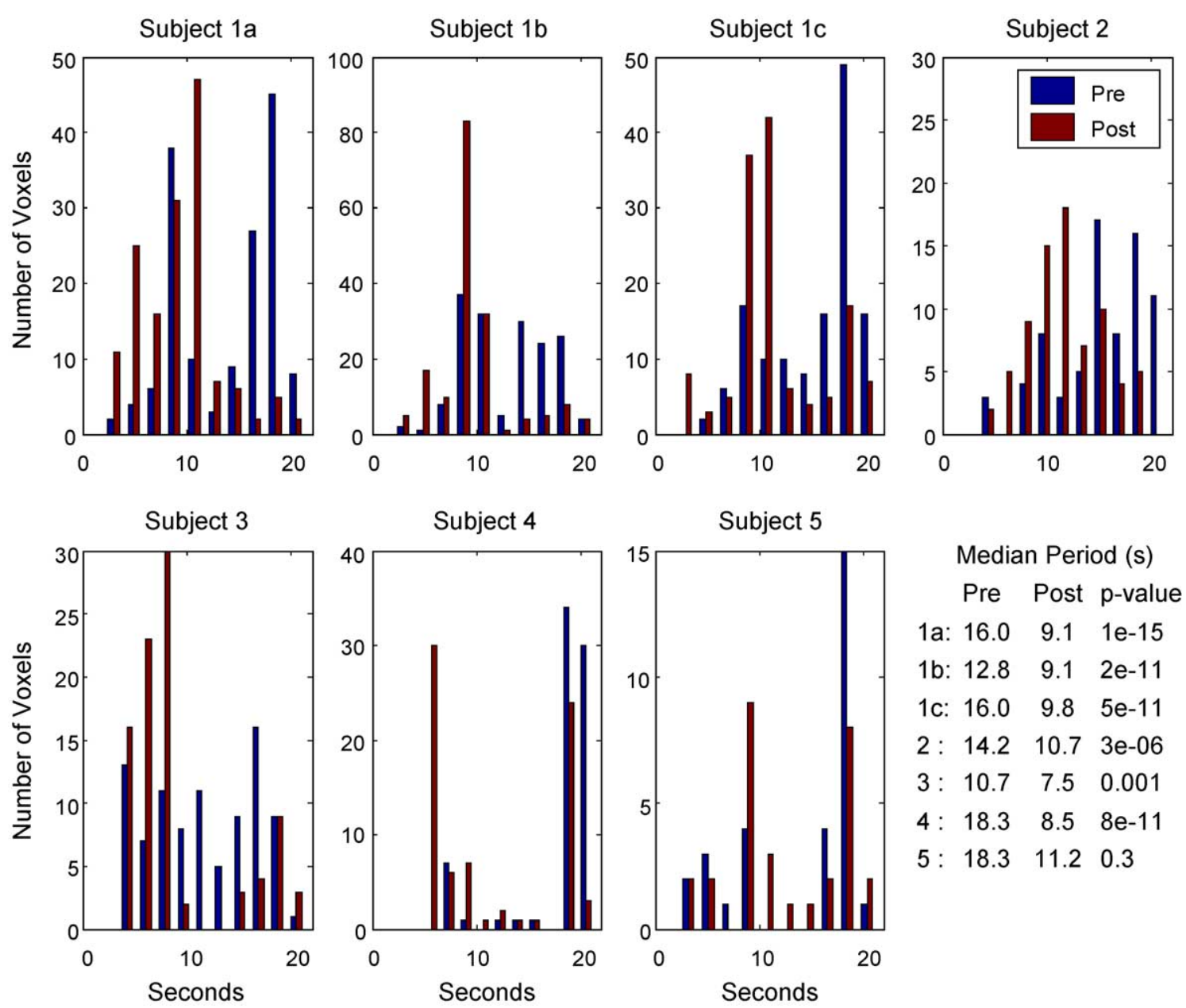

Fig. 9. Histograms of the peak periods observed in the poststimulus portion of the periodic single trial responses. The table shows the median peak period for each subject and condition (predose and postdose) and the $p$-value as computed with a Wilcoxon signed rank test.

dominant postdose peak in the histograms at around $10 \mathrm{~s}$. The median postdose peak period across all subjects showing a significant decrease was $9.1 \mathrm{~s}$. For the block responses, all subjects except for subject 2 showed a significant $(p<0.002)$ caffeinerelated decrease in the median peak period, with a median postdose peak period across all significant subjects of $9.5 \mathrm{~s}$. Subjects 1,3 , and 5 show a dominant postdose peak in the histograms at around $10 \mathrm{~s}$, except for the first day for subject 1 (data set 1a). The probability values reported were obtained with a Wilcoxon signed rank test. Analysis with a paired $t$ test yielded similar results and did not change the determination of which subjects showed a significant effect.

The reproducibility of the caffeine-induced dynamic changes for subject 1 is demonstrated in a qualitative fashion by the ROI averaged curves of Fig. 11. The postdose responses across the 3 days exhibit a strong similarity with pronounced oscillations that are not as obvious in the predose responses. These oscillations are consistent with the peak period data discussed above. As shown in Fig. 6, significant $(p<0.05)$ decreases in $\mathrm{TA}_{10}, \mathrm{~T}_{50}, \mathrm{TA}_{50}$, FWHM, and time to peak of the single trial responses were observed on all 3 days. The peak amplitude showed a significant increase on days 1 and 10 and a significant decrease on day 8, primarily due to an increase in the mean peak amplitude of the predose response on day 8. As shown in Fig. 7, significant decreases in $\mathrm{T}_{10}, \mathrm{TA}_{10}, \mathrm{~T}_{50}, \mathrm{TA}_{50}$, and time to peak of the block responses were observed on all 3 days.

Measurements of baseline CBF are shown in Table 1. All subjects exhibited a significant $(p<0.004)$ caffeine-induced decrease in $\mathrm{CBF}$ with a mean decrease over all subjects of $24 \%$ (treating the average of subject 1 as one sample point).

\section{Discussion}

\section{Changes in BOLD dynamics}

Caffeine alters the temporal dynamics of the BOLD response, leading to significant decreases in $\mathrm{TA}_{50}$ and the time to peak for both single trial and block responses and a significant decrease in the amplitude of the poststimulus response for the single trial responses and $\mathrm{T}_{50}$ for the block responses. In addition, other parameters were found to show significant decreases in many of the subjects. The shapes of the block responses exhibit a qualitative difference that is reflected in the significant differences in time to peak. The postdose block response rapidly rises to its peak and decays gradually during the stimulus period, whereas the predose block response shows an initial rapid rise followed by a slow approach to its peak value, which is obtained after the cessation of 

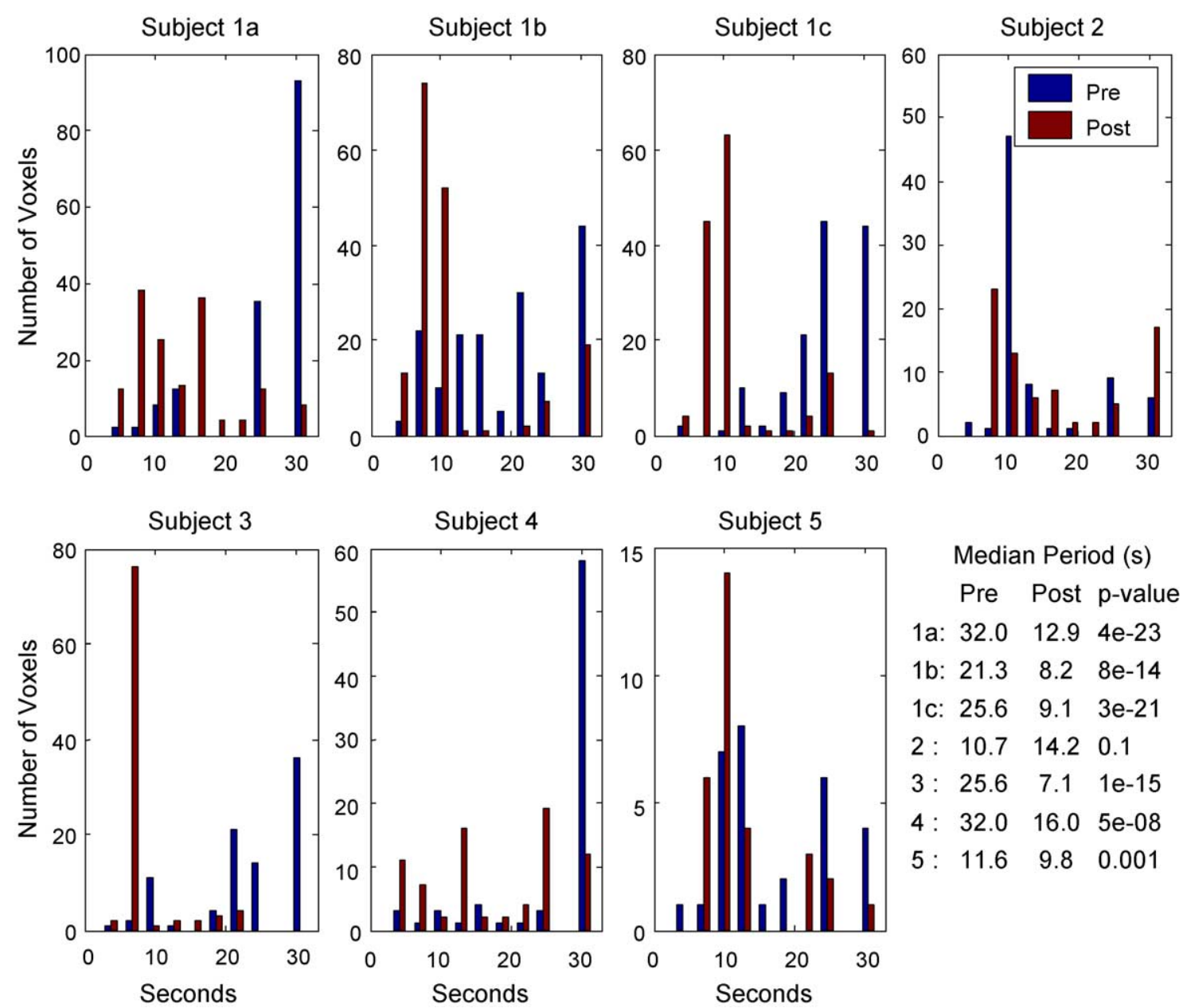

Fig. 10. Histograms of the peak periods observed in the poststimulus portion of the block responses. The table shows the median peak period for each subject and condition (predose and postdose) and the $p$-value as computed with a Wilcoxon signed rank test.

stimulus. Caffeine also appears to enhance oscillations in the poststimulus region of the responses. Reproducibility of the effects was demonstrated by the repeated measures on subject 1 .

The observed changes in temporal dynamics are similar to those observed with hypocapnia (Cohen et al., 2002; Kemna and Posse, 2001) and hyperoxia (Kashikura et al., 2001). In particular, the group average single trial responses shown in Fig. $3 \mathrm{~b}$ show a strong resemblance to the average single trial responses shown in Fig. 4 of Cohen et al. (2002). In that study, hypocapnia resulted in an approximately $7 \%$ increase in the BOLD signal change versus the $12 \%$ increase shown in Fig. 1 of this paper. These values differ from the $26 \%$ increase in the amplitude of the visual BOLD single trial response with caffeine reported in Mulderink et al. (2002). However, the authors of that study also report that large standard deviations were observed within the group of subjects. Indeed, we observed significant variability in the amplitudes of the individual subject ROI averaged single trial responses and found no significant group difference in the predose and postdose peak amplitudes.

Stimulus-related oscillations such as those observed in this study have not been widely reported, possibly due to the fact that the oscillations may be greatly attenuated when the responses are averaged over subjects. For example, the hyperoxic visual BOLD response shown in Kashikura et al. (2001) shows a hint of an oscillation with a period of $7 \mathrm{~s}$ but is not discussed in the text. Interestingly, the period of the oscillations is similar to the 10 -s period of the spontaneous background vasomotion observed in the brain and other organs (Mayhew et al., 1996; Mitra et al., 1997; Nilsson and Aalkjaer, 2003; Obrig et al., 2000). The background oscillations are enhanced by reduced perfusion (Nilsson and Aalkjaer, 2003) and decreased by elevated perfusion (Obrig et al., 2000). This dependence on the baseline CBF is similar to what we observe for the stimulus-induced oscillations and suggests a possible shared mechanism for both types of oscillations.

\section{Baseline $C B F$}

A significant decrease in baseline CBF was observed in all subjects, with individual subject percent decreases ranging from $17 \%$ to $40 \%$ and an overall group average decrease of $24 \%$. Positron emission tomography (PET) studies have measured whole-brain decreases of approximately $30 \%$ with a 250 -mg dose of caffeine (Cameron et al., 1990). Field et al. (2003) measured the CBF decrease with a 250-mg caffeine dose using an arterial spin labeling MRI method. They reported a mean decrease of $23 \%$ in posterior gray matter, with some subjects showing a decrease as large $37 \%$. Using an arterial spin labeling sequence similar to the one used in this study (PICORE QUIPSSII), Mulderink et al. 

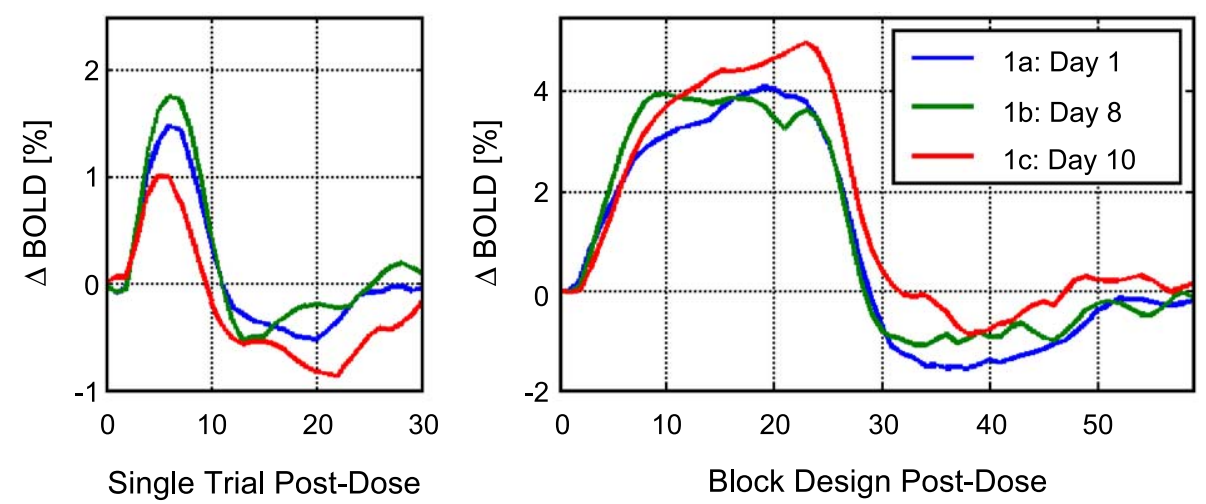

Single Trial Post-Dose
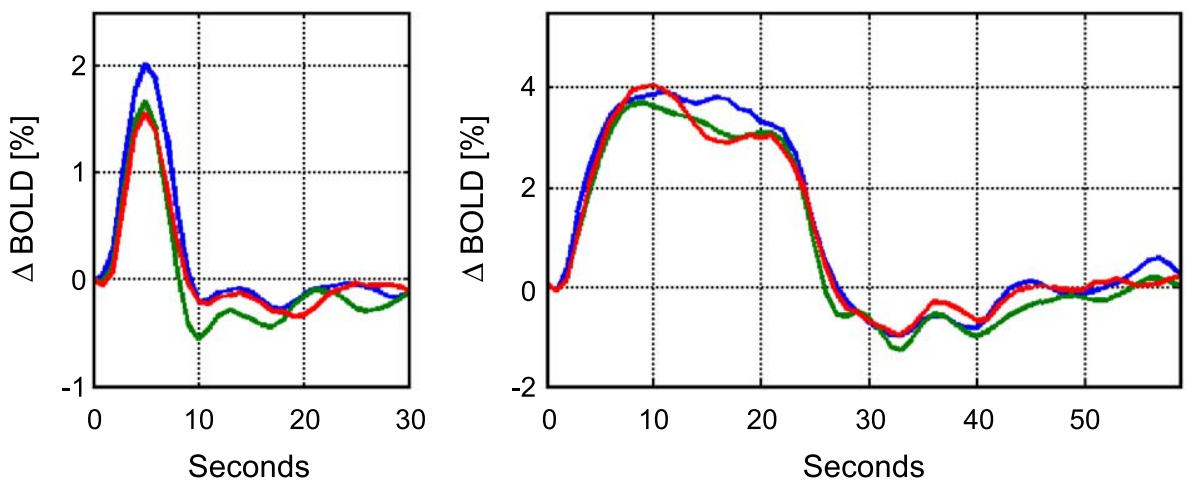

Fig. 11. Predose and postdose single trial and block BOLD responses for subject 1 data acquired on days 1 , 8 , and 10 .

(2002) report a mean $\mathrm{CBF}$ decrease of $11 \%$ in visual cortex; however, these values are difficult to assess because the sequence parameters and analysis are not described in detail. On the whole, our measurements of decreases in baseline CBF are consistent with previous work. A possible source of errors in our measurements comes from using the $M_{0}+T_{2} *$ EPI images as a rough estimate of $M_{0 A} \exp \left(-\mathrm{TE} / T_{2 B} *\right)$. This potential source of error can be addressed in future studies with short echo time acquisition sequences and the use of images of large blood vessels (e.g., sagittal sinus) to better estimate $M_{0 A}$.

\section{Limitations of the experimental protocol}

A limitation of the experimental protocol used in the present study is the insertion of a period between predose and postdose sections during which the subject emerged from the scanner to ingest the caffeine dose. This protocol was adopted so as to minimize subject discomfort and movement given the long duration of the total imaging procedure (approximately $3 \mathrm{~h}$ without a break) and is similar to that used in a previous caffeine fMRI study (Mulderink et al., 2002). In another study, the predose and postdose data were acquired on different days (Laurienti et al., 2002, 2003)

Because of the nature of the protocol, image registration was required to align the predose and postdose imaging volumes. One source of error in the registration process is the differential warping of the EPI images due to differences in both the shimming process and patient head position between the predose and postdose sections. This differential warping is somewhat minimized by relative uniformity of the static magnetic field in visual cortex, as compared to regions of the brain closer to the sinus cavities.
Misregistration of the predose and postdose volumes would tend to reduce the significance of the voxelwise comparisons. Thus, although we report significant differences using paired $t$ tests in the present study, it is likely that improvements in the protocol would improve the significance of the results.

Future studies would clearly benefit from changes to the protocol, such as the administration of caffeine in a liquid form so as to obviate the need to remove the subject from the scanner (Dager et al., 1999) and the use of a bite-bar to minimize patient movement during the scan. Administration of caffeine while the subject is in the scanner would also allow for the measurement of dynamic changes that occur as caffeine is being absorbed (Dager et al., 1999). Despite the limitations of the current protocol, the demonstration of significant changes in this study is encouraging for future studies that may need to use a similar type of protocol to study drugs that require a longer period for absorption or to follow the effect of a drug over many days or different conditions (e.g., pre- and postsurgery).

\section{Variability of the response}

While all subjects showed an effect of the caffeine dose, there was considerable intersubject variability in the responses as shown by the scatter plots and histograms in the Results section. This is not unexpected, since differences in age, caffeine usage, and body metabolism may all affect the degree to which the caffeine affects the cerebrovascular system. For example, prior caffeine use has been shown to modulate the magnitude of the BOLD signal, presumably due to upregulation of adenosine receptors (Laurienti et al., 2002). In addition, the half-life of caffeine has been shown to depend on factors such as the usage of nicotine or oral contra- 
ceptives (Fredholm et al., 1999), so that different rates of peripheral caffeine metabolism may also contribute to the observed variability. Intersubject variability of the predose responses is also apparent in the data and consistent with previous studies reporting significant intersubject differences in BOLD response (Aguirre et al., 1998; D’Esposito et al., 2003).

Aguirre et al. (1998) have noted that repeated scans in the same subject show much less variability than intersubject scans. It is interesting to note that, from a qualitative viewpoint, the postdose responses from the repeated scans of subject 1 show much less variability than the corresponding predose responses. A possible explanation is that the caffeine dose drives the cerebrovascular system to roughly the same state in each scan session, so that the response to stimulus is more similar across sessions, whereas the variability in predose responses may be related to uncontrolled variables, such as the daily variations in the baseline responsiveness of the vasculature.

\section{Dependence of dynamics on baseline $C B F$}

Why does a decrease in baseline CBF speed up the BOLD response? As Cohen et al. (2002) have remarked, the observed changes are perplexing, since a decrease in baseline CBF might be expected to correspond to reduced blood velocities and therefore lead to a slower dynamic response. This is consistent with the assumption of laminar flow in which the mean velocity decreases with the flow rate because the resistance increases faster than the cross-sectional area of the vessel decreases (Fung, 1997).

Since the BOLD response depends on the CBF response, it is useful to consider whether the dynamics of the CBF response are also sensitive to the baseline CBF. Laser Doppler flow studies in rats have shown that the $\mathrm{CBF}$ response slows down significantly with an elevated baseline $\mathrm{CBF}$ due to hypercapnia (Ances et al., 2001; Bakalova et al., 2001; Matsuura et al., 2000a) and speeds up slightly with decreased baseline $\mathrm{CBF}$ due to either hypocapnia (Matsuura et al., 2000a) or hyperoxia (Matsuura et al., 2000b, 2001). An arterial spin labeling study in rats has reported similar results (Silva et al., 1999). Local field potentials are relatively unaffected by hyperoxia or hypercapnia (Matsuura et al., 2000b), indicating that the observed effect is primarily vascular. Taken together, these results strongly suggest that the observed changes in BOLD dynamics reflect a direct dependence of the CBF dynamics on baseline $\mathrm{CBF}$.

Further evidence comes from transcranial Doppler measurements of middle cerebral artery blood flow velocities. Aaslid et al. (1989) measured the rate of dynamic autoregulation, defined as the change of cerebrovascular resistance per unit time after a step decrease in blood pressure, under normocapnic, hyopcapnic, and hypercapnic conditions. They found that the rate was inversely proportional to the level of carbon dioxide and hence baseline CBF. Subsequent studies have confirmed these findings (Aaslid et al., 1991; Birch et al., 1995; Blaha et al., 2003). Although these studies specifically address dynamic autoregulation with respect to pressure changes in the larger cerebral arteries, it is not unreasonable to expect that the biomechanics governing the autoregulation of the larger vessels may share common features with the biomechanics of smaller arterioles involved in neuronally activated CBF responses. In other words, the mechanism by which baseline vascular tone affects the evoked dynamics may be similar for both pressure-induced and neurally induced changes in $\mathrm{CBF}$.
Preliminary work in our laboratory indicates that a model linking neural activity to changes in arteriolar compliance may account for the observed changes in $\mathrm{CBF}$ dynamics as a function of baseline CBF (Behzadi et al., 2004). In this model, the overall compliance of the arteriole consists of an active component representing vascular smooth muscle and a passive component representing connective tissue. As baseline CBF decreases and the arterioles constrict, the active component plays a more dominant role and leads to a faster response. However, other mechanisms are possible, and additional studies are required to further our understanding of the dependence of the hemodynamic response on baseline CBF.

\section{Conclusions}

Caffeine usage significantly changes the dynamics of the BOLD response and may be partly responsible for the subject-tosubject and day-to-day variability in the shape of the hemodynamic response. The effects of caffeine should therefore be considered in the interpretation of fMRI experiments, especially event-related experiments in which estimates of the hemodynamic response are of interest. It appears that the caffeine-induced changes are due primarily to the decrease in baseline CBF. However, to predict the effects of caffeine in any given subject, other physiological factors, such as the baseline vascular compliance of the subject, must also be considered.

\section{Acknowledgments}

This work was supported by a Biomedical Engineering Research Grant from the Whitaker Foundation and by NIH Grant NS36722.

\section{References}

Aaslid, R., Lindegaard, K.F., Sorteberg, W., Nornes, H., 1989. Cerebral autoregulation dynamics in humans. Stroke 20 (1), 45-52.

Aaslid, R., Newell, D.W., Stooss, R., Sorteberg, W., Lindegaard, K.F., 1991. Assessment of cerebral autoregulation dynamics from simultaneous arterial and venous transcranial Doppler recordings in humans. Stroke 22 (9), 1148-1154.

Aguirre, G.K., Zarahn, E., D’Esposito, M., 1998. The variability of human, BOLD hemodynamic responses. NeuroImage 8, 360-369.

Ances, B.M., Greenberg, J.H., Detre, J.A., 2001. The effects of graded hypercapnia on the activation flow coupling response due to forepaw stimulation in alpha-chloralose anesthetized rats. Brain Res. 911 (1), $82-88$.

Bakalova, R., Matsuura, T., Kanno, I., 2001. Frequency dependence of local cerebral blood flow induced by somatosensory hind paw stimulation in rat under normo- and hypercapnia. Jpn. J. Physiol. 51 (2), 201-208.

Behzadi, Y., Restom, K., Liu, T.T., 2004. Modeling the effect of baseline arteriolar compliance on BOLD Dynamics. Proceedings of the ISMRM 12th Scientific Meeting, Kyoto, Japan, pp. 279.

Birch, A.A., Dirnhuber, M.J., Hartley-Davies, R., Iannotti, F., Neil-Dwyer, G., 1995. Assessment of autoregulation by means of periodic changes in blood pressure. Stroke 26 (5), 834-837.

Blaha, M., Aaslid, R., Douville, C.M., Correra, R., Newell, D.W., 2003. Cerebral blood flow and dynamic cerebral autoregulation during ethanol intoxication and hypercapnia. J. Clin. Neurosci. 10 (2), $195-198$. 
Buxton, R.B., Wong, E.C., Frank, L.R., 1998. Dynamics of blood flow and oxygenation changes during brain activation: the balloon model. Magn. Reson. Med. 39, 855-864.

Cameron, O.G., Modell, J.G., Hariharan, M., 1990. Caffeine and human cerebral blood flow: a positron emission tomography study. Life Sci. 47 (13), $1141-1146$

Cohen, E.R., Ugurbil, K., Kim, S.G., 2002. Effect of basal conditions on the magnitude and dynamics of the blood oxygenation level-dependent fMRI response. J. Cereb. Blood Flow Metab. 22 (9), 1042-1053.

Cox, R.W., 1996. AFNI-software for analysis and visualization of functional magnetic resonance neuroimages. Comput. Biomed. Res. $29,162-173$.

Dager, S.R., Layton, M.E., Strauss, W., Richards, T.L., Heide, A., Friedman, S.D., Artru, A.A., Hayes, C.E., Posse, S., 1999. Human brain metabolic response to caffeine and the effects of tolerance. Am. J. Psychiatry 156 (2), 229-237.

D'Esposito, M., Deouell, L.Y., Gazzaley, A., 2003. Alterations in the BOLD fMRI signal with ageing and disease: a challenge for neuroimaging. Nat. Rev. Neurosci. 4 (11), 863-872.

Engel, S.A., Rumelhart, D.E., Wandell, B.A., Lee, A.T., Glover, G.H., Chichilnisky, E.-J., Shadlen, M.N., 1994. fMRI of human visual cortex. Nature 369, 370 [erratum]:525, 106 [erratum].

Field, A.S., Laurienti, P.M., Yen, Y.-F., Burdette, J.H., Moody, D.M., 2003. Dietary caffeine consumption and withdrawal: confounding variables in quantitative cerebral perfusion studies? Radiology 227, 129-135.

Fredholm, B.B., Battig, K., Holmen, J., Nehlig, A., Zvartau, E.E., 1999. Actions of caffeine in the brain with special reference to factors that contribute to its widespread use. Pharmacol. Rev. 51 (1), 83-133.

Fung, Y.C., 1997. Biomechanics: Circulation. Springer-Verlag, New York.

Gati, J.S., Menon, R.S., Ugurbil, K., Rutt, B.K. 1997. Experimental determination of the BOLD field strength dependence in vessels and tissue.

Handwerker, D.A., Ollinger, J.M., D’Esposito, M., 2004. Variation of BOLD hemodynamic responses across subjects and brain regions and their effects on statistical analyses. NeuroImage 21 (4), 1639-1651.

Kashikura, K., Kershaw, J., Kashikura, A., Zhang, X., Matsuura, T., Kanno, I., 2001. Hyperoxia modified activation-induced blood oxygenation level-dependent response of human visual cortex (V1): an event-related functional magnetic resonance imaging study. Neurosci. Lett. 299 (1-2), $53-56$.

Kemna, L.J., Posse, S., 2001. Effect of respiratory CO(2) changes on the temporal dynamics of the hemodynamic response in functional MR imaging. NeuroImage 14 (3), 642-649.

Laurienti, P.J., Field, A.S., Burdette, J.H., Maldjian, J.A., Yen, Y.-F., Moody, D.M., 2002. Dietary caffeine consumption modulates fMRI measures. NeuroImage 17, 751-757.

Laurienti, P.J., Field, A.S., Burdette, J.H., Maldjian, J.A., Yen, Y.F., Moody, D.M., 2003. Relationship between caffeine-induced changes in resting cerebral perfusion and blood oxygenation level-dependent signal. AJNR Am. J. Neuroradiol. 24 (8), 1607-1611.

Lu, H., Clingman, C., Golay, X., Zijl, P.V., 2003a. What is the longitudinal relaxation time (T1) of blood at 3.0 Tesla. Proceedings of the ISMRM Eleventh Scientific Meeting, Toronto, Canada, pp. 669.

Lu, H., Golay, X., Pekar, J.J., Van Zijl, P.C., 2003b. Functional magnetic resonance imaging based on changes in vascular space occupancy. Magn. Reson. Med. 50 (2), 263-274.
Matsuura, T., Fujita, H., Kashikura, K., Kanno, I., 2000a. Evoked local cerebral blood flow induced by somatosensory stimulation is proportional to the baseline flow. Neurosci. Res. 38 (4), 341-348.

Matsuura, T., Fujita, H., Kashikura, K., Kanno, I., 2000b. Modulation of evoked cerebral blood flow under excessive blood supply and hyperoxic conditions. Jpn. J. Physiol. 50 (1), 115-123.

Matsuura, T., Kashikura, K., Kanno, I., 2001. Hemodynamics of local cerebral blood flow induced by somatosensory stimulation under normoxia and hyperoxia in rats. Comp. Biochem. Physiol., A Mol. Integr. Physiol. 129 (2-3), 363-372.

Mayhew, J.E., Askew, S., Zheng, Y., Porrill, J., Westby, G.W., Redgrave, P., Rector, D.M., Harper, R.M., 1996. Cerebral vasomotion: a 0.1-Hz oscillation in reflected light imaging of neural activity. NeuroImage 4 (3 Pt 1), 183-193.

Mitra, P.P., Ogawa, S., Hu, X., Ugurbil, K., 1997. The nature of spatiotemporal changes in cerebral hemodynamics as manifested in functional magnetic resonance imaging. Magn. Reson. Med. 37 (4), $511-518$

Mulderink, T.A., Gitelman, D.r., Mesulam, M.-M., Parrish, T.B., 2002. On the use of caffeine as a contrast booster for BOLD fMRI studies. NeuroImage 15, 37-44.

Ngai, A.C., Coyne, E.F., Meno, J.R., West, G.A., Winn, H.R., 2001. Receptor subtypes mediating adenosine-induced dilation of cerebral arterioles. Am. J. Physiol, Heart Circ. Physiol. 280 (5), $\mathrm{H} 2329-\mathrm{H} 2335$.

Nilsson, H., Aalkjaer, C., 2003. Vasomotion: mechanisms and physiological importance. Mol. Interv. 3 (2), 79-89.

Obrig, H., Neufang, M., Wenzel, R., Kohl, M., Steinbrink, J., Einhaupl, K., Villringer, A., 2000. Spontaneous low frequency oscillations of cerebral hemodynamics and metabolism in human adults. NeuroImage 12 (6), $623-639$

Press, W.A., Brewer, A.A., Dougherty, R.F., Wade, A.R., Wandell, B.A., 2001. Visual areas and spatial summation in human visual cortex. Vision Res. 41 (10-11), 1321-1332.

Rosen, B.R., Buckner, R.L., Dale, A.M., 1998. Event-related functional MRI: past, present, and future. Proc. Natl. Acad. Sci. U. S. A. 95, $773-780$.

Silva, A.C., Iadecola, C., Kim, S.-G., 1999. The dependence of BOLD and $\mathrm{CBF}$ changes on the $\mathrm{CBF}$ baseline during somatosensory simulation. Proceedings of the Seventh ISMRM Scientific Meeting, Philadelphia, PA, pp. 1736

Silvennoinen, M.J., Clingman, C.S., Golay, X., Kauppinen, R.A., van Zijl, P.C., 2003. Comparison of the dependence of blood R2 and R2* on oxygen saturation at 1.5 and 4.7 Tesla. Magn. Reson. Med. 49 (1), $47-60$.

Teo, P.C., Sapiro, G., Wandell, B.A., 1997. Creating connected representations of cortical gray matter for functional MRI visualization. IEEE Trans. Med. Imag. 16 (6), 852-863.

Wandell, B.A., Chial, S., Backus, B.T., 2000. Visualization and measurement of the cortical surface. J. Cogn. Neurosci. 12 (5), 739-752.

Wong, E.C., Buxton, R.B., Frank, L.R., 1997. Implementation of quantitative perfusion imaging techniques for functional brain mapping using pulsed arterial spin labeling. NMR Biomed. 10, 237-249.

Wong, E.C., Buxton, R.B., Frank, L.R., 1998. Quantitative imaging of perfusion using a single subtraction (QUIPSS and QUIPSS II). Magn. Reson. Med. 39 (5), $702-708$. 